\title{
Pós-fenomenologia e Ciência da Informação: aportes epistêmicos para acesso ao conhecimento
}

\author{
Postphenomenology and Information Science: epistemics contributions to knowledge access
}

\author{
Márcia Feijão de Figueiredo \\ Mestre em Ciência da Informação Instituto Brasileiro de Informação em Ciência e Tecnologia - IBICT. \\ Bibliotecária no Colégio Pedro II, na Unidade São Cristovão. \\ E-mail: marciaffigueiredo@gmail.com
}

\section{Resumo}

\begin{abstract}
Apresenta estudos preliminares sobre o uso da Pós-Fenomenologia na Ciência da Informação, fundamentando o acesso ao conhecimento fornecido pela informação imagética produzida por tecnologias e disponibilizada na $w e b$. Descreve os principais elementos que ajudaram a desenvolver essa escola de pensamento e os autores da Fenomenologia que contribuíram para o seu desenvolvimento teórico, Husserl e Merleau-Ponty. Analisa o autor Don Ihde, um dos mais apontados na literatura sobre Pós-Fenomenologia e sua importância para os estudos que relacionam o acesso ao conhecimento mediado na relação homem-tecnologia-mundo, sobretudo numa perspectiva visual dentro da ciência. Aponta abordagens possíveis para estudos futuros que envolvam o uso de tecnologias de comunicação e informação (TICs) e o compartilhamento de informações imagéticas na web.
\end{abstract}

Palavras-chave: Pós-Fenomenologia. Fenomenologia. Informação imagética.

\begin{abstract}
Presents preliminary studies on the use of Postphenomenology in Information Science, supporting access to knowledge provided by imagetic information produced by different technologies and available on the web. Describes the main elements that helped build this school of thought and the authors of phenomenology who contributed to the development of the theory, Husserl and Merleau-Ponty. Analyses of the author Don Ihde, one of the most mentioned in the literature on Postphenomenology and its importance for studies that relate the mediated access to knowledge in the man-technology-world relationship, especially under a visual perspective in science. Points out possible approaches for future studies involving the use of information and communication technologies (ICTs) and imagetic information sharing on the web.
\end{abstract}

Keywords: Postphenomenology. Phenomenology. Imagetic information.

\section{Introdução}

A importância de estudos com enfoque na epistemologia dentro da Ciência da Informação - CI tem sido ressaltada por diversos autores. González de Gómez (2007) afirma que, "além de constituir um espaço meta-teórico de reflexão e crítica, a epistemologia contribui também para a construção de conhecimentos teóricos, procedimentais e práticos". A edição de um número especial da Journal of Documentation em 2005, onde diversos autores refletem sobre as escolas de pensamento filosófico e a relação com os estudos em Biblioteconomia e 
Ciência da Informação (Library and Information Science - LIS) através de suas contribuições para o desenvolvimento teórico da área, é um reconhecimento dessa necessidade.

Algumas abordagens práticas da CI estão desenvolvendo, como o uso das tecnologias de comunicação e informação (TICs), a web como meio de compartilhamento de informações digitais e, em específico, a mediação da informação imagética. Estas aplicações necessitam de reflexões teóricas para sua justificação epistemológica na CI. Enquanto que o uso de imagens de função epistêmica ocorre há séculos, as mídias e tecnologias digitais são muito recentes e carentes de estudos fundamentados em teorias desenvolvidas na epistemologia.

Para atender a essa demanda que o campo apresenta, este trabalho pretende-se valer de alguns aportes teóricos da Pós-Fenomenologia para compreender o uso de imagens de função informativa nas ciências. Reconhecido este uso como uma aplicação remota, cabe-nos refletir sobre sua expressão histórica e os modelos atuais. Um exemplo muito recorrente de imagens e dispositivos nas ciências no passado foi a utilização da câmera obscura, cujo efeito óptico já "era conhecido na antiguidade, mas foi plenamente descrito pela primeira vez de modo técnico por Al Hazen em 1037” (IHDE, 2004, p. 20), e se constituía de:

[...] uma sala ou uma caixa cercada, com uma pequena abertura - com ou sem uma objetiva - com uma parede branca ou uma tela no lado oposto à abertura, sobre a qual é projetada [invertida e em duas dimensões] uma imagem ou representação de alguns objetos iluminados ou fontes de luz própria de fora da caixa (IHDE, 2004, p. 20).

O que se pode considerar uma novidade nas ciências modernas em produção e acesso a imagem epistêmica são os instrumentos atualmente utilizados que se encontram cada vez mais sofisticados para captura e transmissão de imagem. Por exemplo, o acesso aos recursos imagéticos que ocorrem através da internet e por dispositivos digitais, e o entendimento de que a imagem na web implica a mediação de uma atividade intelectual-perceptiva por dispositivos tecnológicos complexos. Na epistemologia, um dos domínios que investiga o uso dessas tecnologias e de instrumentos para mediação entre o homem e o mundo da vida é a PósFenomenologia, fundamentada na Filosofia da Tecnologia, e Don Ihde um de seus principais autores e fundadores. As questões não abarcadas pela Fenomenologia decorreram no desenvolvimento dessa linha de pensamento, mas Ihde utiliza como pressupostos teóricos os últimos trabalhos de Edmund Husserl, cujo enfoque se dava na Fenomenologia transcendental, e os estudos perceptivos de Maurice Merleau-Ponty, principalmente o relatado no livro Fenomenologia da Percepção (1942) (IHDE, 2008). 
O objetivo deste trabalho é compreender o uso das imagens pela ciência através de dispositivos digitais para "acessar" a informação, e a importância da percepção visual dentro da Pós-Fenomenologia. Para a construção desta reflexão foi necessário passar pelos princípios da Fenomenologia que desencadearam a linha de pensamento em torno das teorias da imagem: os estudos de Husserl e Merleau-Ponty, bem como os autores em CI que abordaram a Fenomenologia. Em seguida os conceitos básicos que caracterizam a Pós-Fenomenologia e o histórico dessa recente escola de pensamento para começar os estudos visualistas desenvolvidos por Ihde, e a relevância da imagem para acesso ao conhecimento. Dentro dos estudos imagéticos na ciência descreve-se a importância dos trabalhos de Da Vinci, Galileu e alguns dispositivos que promoveram o acesso as imagens epistêmicas desde a câmera obscura até os atuais mecanismos utilizados nas pesquisas, como o observatório Chandra, da NASA.

\section{Da Fenomenologia ao uso da Pós-Fenomenologia no acesso a informação imagética: aportes}

Fenomenologia: pressuposto teórico para a pós-fenomenologia

Segundo a etimologia, Fenomenologia (fenômeno + -logia) corresponde a um "tratado sobre os fenômenos ou sobre o estudo comparativo dos fenômenos" (FENOMENOLOGIA, 2010). Trata-se, em linhas gerais, de uma frente de pesquisa muito aberta. "Como tudo o que aparece é fenômeno, o domínio da Fenomenologia é praticamente ilimitado e não poderíamos, pois, confiná-la numa ciência particular” (DARTIGUES, 1992, p. 1). Ferrater Mora (1978, p.110) entende que

Quando na época actual se fala de fenomenologia tende-se a entender por tal a fenomenologia de Husserl. Por este motivo referir-nos-emos exclusivamente à fenomenologia husserliana, entendendo-a como _método e como _modo de ver. Constitui-se o método após a depuração do psicologismo.

John Budd esclarece os motivos que levaram os trabalhos de Edmund Husserl a serem reconhecidos como o foco do movimento da Fenomenologia como epistemologia:

Husserl desenvolveu uma definição particular de fenomenologia e, uma vez que ele estava preparando uma nova ciência da percepção e das essências, ele teve que começar com uma nova concepção do 'Ser'. O cerne da fenomenologia de Husserl, e um ponto chave (ou ponto focal) em programas posteriores, é que a abertura para a descoberta da essência das coisas exige que se ignorem opiniões pré-existentes sobre o mundo (BUDD, 2005, p. 46, tradução nossa). 
A concepção husserliana partiu de uma crítica ao psicologismo, entendendo que é uma ciência empírica que não possui regras lógicas e impossibilitam o acesso ao conhecimento científico. Para que esse acesso venha a ocorrer é necessário que o filósofo apresente uma consciência da intencionalidade, isto é, "toda consciência é 'consciência de'. Portanto, a consciência não é uma substância (alma), mas uma atividade constituída por atos (percepção, imaginação, especulação, volição, paixão, etc)" (HUSSERL, 1980, p. viii). A tarefa filosófica de um fenomenólogo

[...] consiste em descrever os fenômenos, e não explicá-los. A primeira vista pode-se pensar que esses sejam procedimentos parecidos. No entanto, explicar implica interferir no fenômeno, introduzindo nele nossas categorias lógicas. Assim, explicar é um ato artificial, enquanto descrever supõe abordar o fenômeno da perspectiva do homem que o vivencia tal como ele se apresenta a consciência (CARMO, 2000, p. 22).

Autor importante dentro da Fenomenologia, sobretudo na corrente francesa, MerleauPonty era um filósofo que tinha por interesse uma filosofia que tratasse dos problemas de sua época, "incorporando as preocupações contemporâneas das ciências humanas, tornando-se mais concreta e menos reflexiva" (CARMO, 2000, p.10). Segundo Robredo (2007, p.42), o filósofo desenvolve o conceito de 'corpo-sujeito' e reflete sobre a parcialidade existencial de nossa visão das coisas. "Uma das propostas dessa corrente era a de que a filosofia deveria se preocupar com a volta 'as próprias coisas' tal como elas se apresentam a nos no mundo" (CARMO, 2000, p. 11). Trabalhar com algumas características da obra de Merleau-Ponty é de grande importância para o desenvolvimento de estudos na Pós-Fenomenologia, enfoque que situa o uso de tecnologias como elemento mediador entre o sujeito e o mundo, principalmente o domínio intitulado Fenomenologia da Percepção.

Maurice Merleau-Ponty aborda de forma crítica os trabalhos de Husserl sobre o corpo e as percepções que desenvolveu em fase tardia sobre a noção de "mundo-da-vida", além dos estudos sobre o corpo e a percepção. O livro Fenomenologia da Percepção traz a relação de corpos como artefatos, considerando o uso como uma extensão das sensações. Como exemplo, o filósofo afirma que a mão que utiliza uma bengala estende sua percepção até a ponta deste objeto, oferecendo condições ao adepto de realizar uma locomoção melhor. "Esse senso imediato de aptidão corporalmente ampliado através de um artefato, eu denominei mais tarde de uma 'relação corporificada' de humanos-mais-tecnologias" (IHDE, 2004, p. 22). Para Adams (2007, p. 3), o pensamento de Merleau-Ponty funciona como ponte entre a Fenomenologia e a Pós-Fenomenologia. 
Para Verbeek (2008), a Fenomenologia se apresenta como uma alternativa para a ciência, ao descrever a realidade e percebê-la como um método que analisa essa relação com os humanos, abordada por diversos autores de modo distinto: Merleau-Ponty através da percepção, Husserl com a consciência e Heidegger pelo "ser-no-mundo". Em outras palavras, trata-se de perceber uma construção de idéias em torno de conceitos como percepção, imagem, uso, dentre outros. "Como ciência filosófica, a Fenomenologia debruça-se sobre questões originárias desde a própria concepção do que seja o conhecimento até o seu papel como modificador da condição humana" (MARCIANO, 2006, p. 189).

A CI e a Fenomenologia possuem uma interconexão que se realiza por meio da linguagem: enquanto que a linguagem na Fenomenologia é conceituada como origem e expressão do conhecimento, na Ciência da Informação "situa o documento, sua principal fonte de estudo, como veículo do conhecimento codificado e formalizado por meio da linguagem" (MARCIANO, 2006, p. 186). Alguns autores acreditam que a Fenomenologia pode trazer aportes para os estudos de sistemas de informação e na relação com os indivíduos que o utilizam, assim como em produtos e serviços (HJÖRLAND, 2005, p. 158; MARCIANO, 2006, p. 186).

A transformação da informação em conhecimento, terreno comum de estudo da CI, é um dos temas centrais de Husserl. Por exemplo, dentro das experiências de um usuário que busca informação a compreensão é genuína, mas dependente, dentre outras coisas, de um conhecimento prévio e de outras informações. Em concordância com Husserl, Merleau-Ponty agrega aos estudos em CI a possibilidade de junção entre ontologia e epistemologia (BUDD, 2005, p. 47).

Provavelmente a mais importante contribuição da Fenomenologia à CI seja a idéia de que não é possível definir uma percepção e uma observação "puras" do mundo. Sob essa concepção, similar à do realismo crítico, toda observação é dependente de uma teoria e de um contexto, estando continuamente sujeita a reformulações (MARCIANO, 2006, p.187). A idéia de uma mediação permanente entre objeto e conhecedor afeta diretamente os estudos epistemológicos da CI. 
O conhecimento mediado: contribuições da pós-fenomenologia para o acesso a informação imagética

A história da Pós-Fenomenologia, segundo Adams (2007, p. 4), se inicia com Johann P. Arnason publicando um artigo no periódico Thesis Eleven, em 1993, pouco depois que formulou esse conceito. No mesmo ano, Don Ihde publica uma coleção intitulada Postphenomenology: essays in the postmodern context. Suzi Adams (2007, p.4) afirma que o sufixo "pós" acrescentado a Fenomenologia não se refere a um lugar além dessa tradicional corrente de pensamento, mas a novas questões que emergem de tendências não esperadas no terreno da Fenomenologia.

Na Pós-Fenomenologia, a idéia central da Fenomenologia na relação homem-mundo compreendida através da intencionalidade permanece, porém, mediada por uma tecnologia. Rosenberger (2008, p. 64) é o autor que aborda o uso das tecnologias para interpretação de imagem e percebe a Pós-Fenomenologia como um título dado por uma emergente escola de pensamento caracterizada por diferentes posições, combinando tradições da Fenomenologia com o pragmatismo, investigando relações humanas com as tecnologias e dando ênfase às análises de estudos de casos concretos.

Para o desenvolvimento desse trabalho, optou-se por levantar na obra de Don Ihde aportes para o uso das tecnologias e o visualismo nas ciências. Ihde é um filósofo norteamericano que possui diversos estudos no âmbito da Filosofia da tecnologia e recentemente se envolveu com os estudos da filosofia da ciência. O filósofo criou ainda, baseado nesta premissa, um grupo de pesquisa intitulado Tecnoscience Research Group e o dirige desde 1998 (IHDE, 2008, p. 1).

Ihde pode ser considerado um importante mediador da filosofia contemporânea, conectando Fenomenologia com pós-modernismo, Filosofia da tecnologia, Filosofia da ciência e Filosofia continental com Filosofia analítica. A Pós-Fenomenologia desenvolvida pelo filósofo agrega à Fenomenologia conceitos não abordados por Husserl e Merleau-Ponty, dentre outros autores clássicos estudados, na relação da Fenomenologia com a tecnologia e o pragmatismo.

Para Adams (2007, p. 4), a abordagem de Idhe se situa dentro das correntes pósmodernas, focando a percepção, as tecnologias visuais e a ciência. No entendimento de Ihde (2004, p. 20), quando Husserl escreveu o livro A crise na filosofia européia "fez dois 
movimentos que anteciparam vagamente um possível papel para uma Filosofia da tecnologia": o reconhecimento das tecnologias de inscrição ou escritura, que levou a comunicação da humanidade a um novo "patamar" no "mundo-da-vida"; e com a publicação do livro A origem da geometria "sustenta que a geometria surgiu da práxis [praxical activity], uma atividade dentro da cultura material" (IHDE, 2004, p. 21). Observar as práticas de uma ciência abstrata dentro de uma cultura material era inédito no início do século XX e coube a Husserl essa abordagem filosófica.

Para Introna (2011, tradução nossa), Ihde caracteriza quatro tipos diferentes de relação entre "eu-tecnologia-mundo". Assim, Ihde oferece uma taxonomia ou estrutura significativa para dar conta das muitas relações diárias do homem com as tecnologias e pode facilitar "nossas considerações sobre as implicações sociais e éticas da tecnologia da informação".

1. Relações incorporadas ('embodiment' relations) - [eu-óculos]-mundo: A tecnologia é o meio de experiência perceptiva subjetiva no mundo. Exemplos: o uso de óculos, a Lua através de olho nu e por meio de telescópio;

2. Relações hermenêuticas (hermeneutic) eu-[mapa-mundo]: As funções da tecnologia se referem imediatamente a algo além desse mundo. Exemplo: o uso de mapas;

3. Relações alteradas ('alterity' relations) - eu-tecnologia-[mundo]: A tecnologia é experimentada como um ser de outra forma. Exemplos: ícones religiosos e robôs inteligentes;

4. Relações de pano de fundo ('background' relations) - eu-[tecnologia]-mundo: A tecnologia não está diretamente implicada em um processo consciente de compromisso por parte do ator humano. Exemplos: sistemas automáticos de aquecimento central, sistemas de controle de tráfego.

Merleau-Ponty, na concepção de Ihde, se aproximou um pouco mais do que ele chama de "Fenomenologia da técnica" por utilizar exemplos de corporificação humana através de artefatos materiais, como a mulher com chapéu de penas, e a bengala de um cego.

Em termos fenomenológicos, essa relação humano-tecnologia é generalizável - é através dessa incorporação perceptivo-corporal que os instrumentos científicos e musicais também são 'corporificados'; através dela, até mesmo o movimento cinético e o tato tornam-se um 'senso de distância'. Claramente, há aqui implicações para uma filosofia da tecnologia (IHDE, 2004, p. 22).

InCID: R. Ci. Inf. e Doc., Ribeirão Preto, v. 3, n.1, p. 21-35, jan./jun. 2012. 
Apesar das abordagens diversificadas que Ihde oferece à Pós-Fenomenologia buscamos neste trabalho aportes que o filósofo desenvolveu e que abarcam os recentes estudos da CI, as tecnologias e o uso de imagens epistêmicas. Para melhor entendimento três abordagens foram escolhidas: a) o corpo tecnológico, como uma terceira dimensão que perpassa os corpos biológico e social de Merleau-Ponty e Foucault b) o acesso ao conhecimento pelas tecnologias; c) a perspectiva visualista da ciência.

a) O corpo tecnológico, como uma terceira dimensão que perpassa os corpos biológico e social de Merleau-Ponty e Foucault;

O corpo é um objeto de estudo interdisciplinar e diversos campos produzem pesquisas sobre esse tema. Na Pós-Fenomenologia, o enfoque é adotado na compreensão da mediação tecnológica entre o sujeito e o objeto. Em Bodies in Technology (2002) Ihde demonstra como ocorre o funcionamento do corpo mediante aparatos tecnológicos, atuando na extensão dos sentidos. Assim, utiliza os trabalhos de Maurice Merleau-Ponty para analisar o corpo em primeira pessoa - com sensações biológicas - e corpos na perspectiva do observador, culturalmente perceptivo, por Michel Foucault.

Ihde apresenta esses aportes como duas dimensões que constituem um corpo tecnológico: uma baseada na Fenomenologia, que compreende um corpo emotivo e perceptivo no mundo (being-in-the-world), chamado "corpo um" (body one); e um corpo que experimenta o mundo, tendo influências sociais e culturais, intitulado "corpo dois" (body two). A terceira dimensão que atravessa estes dois corpos é a tecnológica (IHDE, 2002, p. xi).

Sobre o "corpo um", Ihde afirma que a característica de Merleau-Ponty é a de um corpo ativo que ele trata tanto como ser pré-conceitual quanto pré-cultural e, sem essa percepção, não ocorrem outras experiências. O desenvolvimento e descrição dessa experiência são feitos na perspectiva de primeira pessoa. O uso do termo "corpo um" é baseado nas experiências que Merleau-Ponty descreve, especialmente no livro Fenomenologia da Percepção (IHDE, 2002, p. 17).

Merleau-Ponty dá importância às incorporações das tecnologias e, quando aborda a bengala de um deficiente visual ou um chapéu de penas feminino, entende que existe a extensão da percepção corporal desse indivíduo (IHDE, 2002, p. 7). Uma das primeiras observações feitas pelo autor sobre o corpo na Fenomenologia vem da ideia que Husserl apresenta sobre o 
pensamento que se liga ao mundo através de seu corpo e não estando mais restrita a consciência. "Assim, para Husserl, a consciência é sempre consciência de algo - consciência intencional - e se define não mais como substância, mas como atividade e abertura" (SCHALCHER, 1986, p. 30).

Em contraste com Merleau-Ponty, Foucault trabalha com corpos descritos e analisados em terceira pessoa, construídos através da cultura. Ihde afirma que um corpo objetivado pelo olhar médico em uma clínica, o corpo do condenado em um regicídio, e todas as formas de disciplina são assimilados como uma característica cultural (IHDE, 2002, p. xi).

As reflexões sobre a percepção e a sensação percebidas pelo corpo, porém, não eram pensadas pelos autores nos estudos sobre as tecnologias. Ihde e Selinger (2004, p. 361) afirmam que o pensamento de Merleau-Ponty sobre a tecnociência conduz no leitor a uma visão limitada da ciência ao mundo da vida. A dimensão tecnológica apresentada por Ihde traz elementos dos dois autores, a percepção e as construções socioculturais, através do uso de tecnologias. Ele afirma que existem percepções contemporâneas que se desenvolveram através de elementos culturalmente novos, como os vídeo-games.

O fenômeno do Nintendo, que enfatiza as ações do olho e da mão, foi a extensão de corpos em tecnologias, que vão desde os jogos de vídeo a cirurgias e é um novo, se restrito, estilo de movimento que está muito longe da atividade física ou esporte de dança, se balé clássico ou moderno (IHDE, 2002, p. 138).

Ihde acrescenta que, anterior a essas observações, a Fenomenologia já abordava o uso de tecnologias como extensão corporal, chamado de 'relação incorporada' (embodiment relation). Exemplos utilizados por Merleau-Ponty é o chapéu de penas-longas, estendendo a sensibilidade até o vão da porta, e o uso de óculos por Heidegger, ampliando a visão do indivíduo. Haraway (2006, p. 176), em concordância com Ihde, afirma que o uso de tecnologias vai além da mediação, funcionando como órgãos, plenos de direito, encarnados no corpo.

b) O acesso ao conhecimento pelas tecnologias;

Nenhum conhecimento científico de sua forma, estrutura, comportamento etc. é possível por completo, exceto através da mediação da tecnologia. Uma vez que um fenômeno está além do alcance face-a-face, pela distância, tamanho ou estrutura, somente é possível a evidência instrumentalmente mediada (IHDE, 2004, p. 25, grifo do autor). 
O acesso ao conhecimento científico e sua relação com a tecnologia é abordado por Ihde através de três modalidades, tendo por base a Filosofia da tecnologia: a) o conhecimento sobre as tecnologias, em que a ênfase de estudos é o modo como a máquina é feita e quais são suas funcionalidades. Geralmente a base para esses estudos é o conhecimento advindo do engenheiro e do tecnicista; b) o conhecimento tecnológico é o teórico, onde os estudos sobre os princípios e leis da física, química e da eletricidade oferecem às tecnologias o desenvolvimento de suas capacidades. Esses estudos se desenvolvem através do conhecimento de cientistas e de engenheiros cientistas; c) o conhecimento que se desenvolve através das tecnologias, no qual o uso das tecnologias é requerido pela ciência para que esta possa se desenvolver (IHDE, 1997, p.73).

A última modalidade, que trata de um saber tecnologicamente mediado, é o foco da pesquisa de Don Ihde (1997, p. 73). A Pós-Fenomenologia entende que as tecnologias se situam entre o homem e o mundo colaborando e aplicando a ciência como elemento constituinte das práticas científicas para a construção das ciências modernas. Ele também acredita que boa parte das ciências contemporâneas possui dependência do uso de instrumentos para o seu desenvolvimento. "A ciência opera fazendo observações por meio de instrumentos - e eu afirmo que a ciência tem operado dessa maneira pelo menos desde Galileu” (IHDE, 2004, p. 24).

Para Ihde (1997, p.74; 2009, p. 458), Galileu é um exemplo de uso corporificado das tecnologias nas ciências modernas que possui consciência da importância das lentes telescópicas em seus trabalhos. As tecnologias ópticas na ciência moderna ampliaram as trajetórias visualistas do Renascimento, “inventadas" através dos desenhos técnicos de Leonardo da Vinci. Galileu optou por desenvolver a ciência com a visão instrumentalizada em vez de observar seus objetos através do olhar desnudo. Apesar das melhorias apresentadas pelo uso de instrumentos que ampliavam a visão, houve conclusões prematuras nos estudos de Galileu por causa das limitações desses mesmos instrumentos. A aura em torno das estrelas, por exemplo, que Galileu descreveu em seus trabalhos, era um efeito do telescópio, que foi descoberto apenas após sua morte (IHDE, 1997, p. 74).

A Pós-Fenomenologia acredita que o resultado da aquisição de conhecimentos através de instrumentos altera tanto o sujeito que observa o mundo instrumentalizado como o objeto de observação, através do corpo e da reflexão (IHDE, 1997, p. 75). As mediações tecnológicas afetariam tanto as práticas da ciência como as representações sobre as ciências. Dentro dos 
estudos filosóficos, a instrumentação óptica de Galileu possui uma relação histórica com os princípios da epistemologia moderna. O uso da câmera obscura por Descartes, por exemplo, faz compreender que ele continuou a trajetória visualista iniciada na Renascença e os estudos de Galileu, apresentando a visão como um modelo de acesso ao conhecimento de forma passiva, recebendo estímulos através do instrumento no corpo.

c) A perspectiva visualista da ciência

Para Ihde (2009, p. 453-454) existe um crescente reconhecimento do que ele chama de ‘visualismo tecnocientífico' (technoscience`s visualism), ou seja, uma prática das ciências e das engenharias em utilizar predominantemente e de forma privilegiada a visão através de instrumentos e deixando de forma secundária as demais percepções corporais. Dentro dessas práticas há também a redução da visão a um estilo, objetivado, que exclui as demais formas de visualizar dentro das ciências. A redução das percepções, a visão e uma nova redução dentro da visão ao objetivismo são chamadas de dupla redução (double reduction). A visão objetivada em questão é mais específica, por excluir o que chama de variações imaginativas (imaginative variations) produzidas pela mente, e o que Ihde também chama de Gedankenexperimenten. Hoje a interpretação e a ilustração são exemplificadas através da proliferação de tecnologias de imagem (imaging technologies), muito utilizadas nas ciências naturais.

Introna (2011) ao descrever as categorias de Ihde para as relações entre o homem e o mundo através da tecnologia, exemplifica o termo hermenêutico da mediação tecnológica nas ciências:

Embora eu possa corrigir o meu foco sobre o texto ou o mapa, o que eu realmente vejo (o encontro) não é o mapa em si, mas sim de imediato e, simultaneamente, o mundo que se refere, e a paisagem sugerida nos símbolos. Neste caso, a transparência da tecnologia é hermenêutica e não perceptual (INTRONA, 2011, tradução nossa)

Para embasar a afirmação de que o visual na ciência é um hábito cultural, Ihde o demonstra através de fatos históricos. O primeiro momento das ciências modernas, em que o uso do visualismo para apresentações científicas foi ressaltado ocorreu através das ilustrações de Leonardo da Vinci, pois a anatomia descritiva passou a ser feita através dos desenhos de tendões, músculos e veias, em vez de utilizar o olfato e o tato do pesquisador, em torno de 1540 D. C. Na mesma época, aumentou o uso da câmera obscura através de Alberti (1437), reduzindo o tridimensional para bidimensional.

InCID: R. Ci. Inf. e Doc., Ribeirão Preto, v. 3, n.1, p. 21-35, jan./jun. 2012. 
Apesar de alguns instrumentos ópticos terem sido comercializados por Paolo Scarpi, foi Galileu Galilei quem trouxe à ciência o instrumento para a visualização na ciência. Esse foi o segundo momento histórico do visualismo, onde um instrumento passa a ser utilizado como mediador entre o sujeito e o objeto, chamado por Ihde de Realismo Instrumental (Instrumental Realism). O terceiro momento foi também o que foi aceito de forma rápida pela comunidade: a fotografia. Em 1840, uma fotografia da lua minguante caracterizou o uso de máquinas fotográficas pela ciência e, para Ihde, assim como a câmera obscura, reduziu o objeto a uma imagem isomórfica e realista em uma chapa fotográfica.

Ihde considera que o momento atual da imagem serve como uma analogia de uma câmera obscura pós-moderna, onde existe a expectativa de diversos usos das tecnologias pela ciência, mas, mas continua limitando-se a visualização (IHDE, 2008, p. 467). O autor acredita que o visualismo, perante outros modos de percepção como o tato e o paladar, que foram muito utilizados na ciência, está em posição confortável, mas essas outras percepções podem trazer resultados mais completos, se utilizando todas as possibilidades que um corpo pode perceber. Ao relacionar a Fenomenologia com as tecnologias, apresenta um exemplo contemporâneo e prático sobre o acesso a um conhecimento obtido através da visão em meio científico:

Eis a parte do conhecimento: aproximadamente no centro da constelação $C r a b$ Nebula há um pulsar rapidamente giratório de cujos eixos estão sendo emitidos dois jatos de radiação raio-x que estão disparando para fora do pulsar quase na velocidade da luz (IHDE, 2002, p. 23-24, tradução nossa).

\section{Considerações Finais}

Desse modo, as tecnologias, os instrumentos, tornam o fenômeno disponível, mas por meio da sua transformação em uma imagem (ou dado) perceptível por humanos corporificados (ou intérpretes)" (IHDE, 2004, p. 25).

A Pós-Fenomenologia, uma nova escola de pensamento que estuda as mediações tecnológicas que ocorrem entre o homem e o mundo, possui suas bases filosóficas na Fenomenologia utilizando textos de Husserl, Merleau-Ponty e Heidegger. Don Ihde é um dos filósofos que fazem parte dessa linha e utiliza, além dos clássicos exemplos científicos de mediação tecnológica, exemplos contemporâneos que embasam sua teoria. É um autor que consegue conciliar o uso de instrumentos na ciência para mediar o acesso a conhecimentos com a predominância do uso de imagens no meio científico em comparação a outras percepções que caíram em desuso com o passar dos anos, o olfato e o tato, por exemplo. 
A primeira observação do Don Ihde é a corporificação de instrumentos utilizando a percepção em primeira pessoa desenvolvida por Merleau-Ponty, e a construção social e cultural dos trabalhos de Foucault, elementos intrínsecos apresentados no corpo humano que decorre em uma terceira dimensão, tecnológica. Esse corpo tecnológico não seria possível sem os elementos apresentados por esses filósofos, visto que a utilização de tecnologias pelo homem perpassa pela percepção e as influências sofridas pela cultura que vivencia.

A segunda observação feita por Ihde é o uso das tecnologias nas ciências como mediador do conhecimento, cujo instrumentos cada vez mais sofisticados permitem acesso a informações que sequer eram cogitadas. Para isso cita Galileu e o telescópio, que permitiu ao cientista observar fatos no universo que não seriam visíveis a olho nu, e que podemos estender a aparelhos de raios $\mathrm{x}$, microscópios e outros instrumentos que ampliam a visão e gera imagens que se tornam conhecimento. Aliás, a visão amplificada por meios tecnológicos é a ultima colocação do autor utilizada em nossas observações: a ciência, predominantemente, utiliza a percepção visual para acessar e produzir conhecimentos.

Segundo Ihde, essa predominância é cultural e se consolidou com o tempo através de diversos fatos que ocorreram na produção da ciência: a descoberta do efeito ótico, a câmera obscura, os desenhos científicos de Leonardo da Vinci, o telescópio de Galileu e uso da fotografia para reproduzir fenômenos. Ihde (1991, p. 79) admite que a percepção científica seja um modo altamente especializado de percepção, e a observação científica se encontra sempre entre a percepção e a mediação tendo a tecnologia como interface.

Os aportes Pós-Fenomenológicos neste trabalho procuraram demonstrar um olhar diferente para o uso de tecnologias nas ciências: a produção e o acesso ao conhecimento mediado pelas máquinas. O entendimento que existe uma ampliação dos sentidos através da mediação tecnológica, principalmente pelo visual, conferiu maior importância de se aceitar as tecnologias como parte dos processos de produção do conhecimento, pois a ciência não teria se desenvolvido nem alcançado novos patamares se não houvesse colaboração desses meios durante a investigação.

$\mathrm{Na}$ medida em que atravessa conceitos centrais do pensamento informacional, como mediação, acesso e percepção, a Pós-fenomenologia pode oferecer aportes teóricos para embasar estudos epistêmicos na CI que contemplam o uso de tecnologias para acessar conhecimento e compreender como essa apropriação colabora para a visualização de imagens, cuja função informativa vem se multiplicando com a evolução de meios como a web e as 
tecnologias de comunicação e informação (TICs), além da proliferação de outros aparatos científicos, como máquinas utilizadas na medicina, astronomia e outros campos de conhecimento.

Conclui-se que a Ciência da Informação possui a crescente preocupação com o mundo mediado por aparatos tecnológicos e a pós-fenomenologia, como escola de pensamento que focaliza seus esforços na compreensão da relação "homem-[tecnologia]-mundo", pode contribuir na abordagem de viés epistêmico nas pesquisas relacionadas com a busca da informação, sobretudo das imagens de viés informativo para o usuário.

\section{Referências}

ADAMS, S. Introduction to post-phenomenology. Thesis Eleven, Thousands Oaks, n. 90, p. 3-5, Aug. 2007.

BUDD, J. M. Phenomenology and information studies. Journal of Documentation, London, v. 61, n. 1, p. $44-59,2005$.

CARMO, P. S. Merleau-Ponty: uma introdução. São Paulo: Educ, 2000. (Coleção Trilhas).

DARTIGUES, A. O que é a fenomenologia? 3. ed. São Paulo: Moraes, 1992.

FENOMENOLOGIA. In: CALDAS Aulete dicionário digital. Rio de Janeiro: Lexikon, 2010.

FERRATER MORA, J. Fenomenologia. In: Dicionário de filosofia. Lisboa: Dom Quixote, 1978.

GONZÁLEZ DE GÓMEZ, M. N. Novas configurações do conhecimento e validade da informação. In: ENCONTRO NACIONAL DE PESQUISA EM CIENCIA DA INFORMACAO, 8, 28-31 out. 2007, Salvador. Anais... Salvador: UFBA/PPGCI; ANCIB, 2007. Disponível em: <http://www.enancib.ppgci.ufba.br/> Acesso em: 8 fev. 2010.

HARAWAY, D. J. Crittercam: compounding eyes in naturecultures. In: SELINGER, E. (Ed.) Postphenomenological: a critical companion to Ihde. New York: State University of New York, 2006. Cap. 12.

HJØRLAND, B. Afterword: comments on the articles and proposals for further work. Journal of Documentation, v. 61, n. 1, p. 44-59, 2005.

HUSSERL, E. Vida e obra. In: Investigações lógicas: sexta investigação. São Paulo: Abril Cultural, 1980.

IHDE, D. Bodies in technology. Minneapolis: Minnesota Press, 2002. (Electronic Mediations, 5). 
2009 .

. From da Vinci to CAD and beyond. Synthese, Dordrecht, v. 168, n.3, p. 453-467,

. Incorporando a matéria: fenomenologia e filosofia da tecnologia. Política \&

Trabalho: revista de ciências sociais, João Pessoa, n. 21, p. 19-30, out. 2004. $1-9,2008$.

Introduction: postphenomenological research. Human Studies, Dordrecht, v. 31, p.

Instrumental realism: the interface between philosophy of science and philosophy of technology. Bloomington: Indiana University Press, 1991.

The structure of technology knowledge. International Journal of Technology and Design Education, Dordrecht, v. 7, p. 73-79, 1997.

. ; SELINGER, E. Merleau-Ponty and epistemology engines. Human Studies, Dordrecht, v. 27, p. 361-376, 2004.

INTRONA, L. Phenomenological Approaches to Ethics and Information Technology. In.: ZALTA, E. N. (ed.) The Stanford Encyclopedia of Philosophy. Stanford, CA: Center for the Study of Language and Information, Stanford University, Summer 2011. Disponível em: $<$ http://plato.stanford.edu/archives/sum2011/entries/ethics-it-phenomenology/>. Acesso em: 27 abr. 2011.

MARCIANO, J. L. P. Abordagens epistemológicas à Ciência da Informação: fenomenologia e hermenêutica. TransInformação, Campinas, v. 18, n. 3, p. 181-190, set./dez. 2006.

Disponível em: <http://revistas.puc-campinas.edu.br/transinfo/viewarticle.php?id=180> . Acesso em: 06 nov. 2011.

ROBREDO, J. Filosofia da ciência da informação ou ciência da informação e filosofia? In:

TOUTAIN, L.M. B. B. (Org.) Para entender a Ciência da Informação. Salvador:

EDUFBA, 2007. p. 35-74. Disponível em: <http://www.repositorio.

ufba.br/ri/handle/ufba/145>. Acesso em: 06 nov. 2011.

ROSENBERGER, R. Perceiving other planets: bodily experience, interpretation, and the mars orbiter camera. Human Studies, v. 31, n. 1, p. 63-75, 2008.

SCHALCHER, M. G. Fenomenologia e linguagem em Merleau-Ponty. 1986. $134 \mathrm{f}$. Dissertação (Mestre em Filosofia) - Instituto de Filosofia e Ciências Sociais, Universidade Federal do Rio de Janeiro, Rio de Janeiro, 1986.

VERBEEK, P. P. Obstetric ultrasound and the technological mediation of morality: a postphenomenological analysis. Human Studies, v. 31, n. 1, p. 11-26, 2008. 